\title{
LDA+DMFT approach to electronic structure of sodium metal
}

\author{
L. Craco ${ }^{1}$ and S. Leoni ${ }^{2}$ \\ ${ }^{1}$ Instituto de Física, Universidade Federal de Mato Grosso, 78060-900, Cuiabá, MT, Brazil \\ ${ }^{2}$ School of Chemistry, Cardiff University, Cardiff, CF10 3AT, United Kingdom
}

(Received 6 June 2019; revised manuscript received 20 August 2019; published 27 September 2019)

\begin{abstract}
Based on local density approximation plus dynamical mean-field theory (LDA+DMFT) calculations, we revisit the long-standing quasiparticle band narrowing problem of sodium metal. Using an on-site Coulomb interaction $U$ derived from angle-resolved photoemission spectroscopy mass enhancement, we can describe various properties of this weakly correlated electron system. Intrinsic self-energy corrections lead to a LandauFermi liquid state with many-particle coherence and dynamical spectral weight transfer relevant to electronic structure and scattering rates of alkali metals.
\end{abstract}

DOI: 10.1103/PhysRevB.100.115156

\section{INTRODUCTION}

Sodium traditionally has been considered to be one of the best realizations of nearly-free-electron gas that can be found in three-dimensional solid state crystals. Its electronic structure has been studied for more than 80 years. [1] Historically, angle-resolved photoemission spectroscopy (ARPES) experiments by Plummer et al. [2,3] pointed out that the quasiparticle dispersion curve for the occupied bands of $\mathrm{Na}$ is remarkably narrowed in comparison with the usual value obtained from band-structure calculations [1]. This remarkable result was corroborated by x-ray absorption (XAS) [4] experiments showing a substantially smaller unoccupied bandwidth than that predicted by band-structure calculations: This discrepancy is linked to the fact that spectroscopy measurements probe the excitation spectra while band-structure calculations (based, for example, on local density approximation, LDA) [5] describe ground-state properties [3]. Motivated thereby, a number of studies [3,6-8] have been conducted to understand the quasiparticle band narrowing of $\mathrm{Na}$ in terms of self-energy corrections due to many-body effects. However, a conclusive answer to this problem has not yet been found in spite of many different efforts [9-14].

In this paper, we report correlated band-structure calculations for the contribution of self-energy corrections arising from local Coulomb interactions. Namely, we employ the LDA plus dynamical mean-field theory (LDA+DMFT) formalism [15] for the multiband, multiorbital (MO) problem of $\mathrm{Na}$. We use the iterated perturbation theory (IPT) impurity solver $[16,17]$ to DMFT, showing the role played by the local Coulomb repulsion to intrinsic bandwidth reduction of both valence and conduction band states of $\mathrm{Na}$. Our results attest that the electronic states near the Fermi energy $\left(E_{F}\right)$ probed by photoemission and inverse photoemission experiments $[2-4,10]$ are narrowed under the influence of sizable on-site Coulomb interactions responsible for low-energy quasiparticle excitations in solid Na.

We shall first point out here the study by Dolado et al. [12], which finds only small momentum dependence on calculated lifetimes of $\mathrm{Na}$ at different $k$-directions and approximately quadratic energy scaling characteristic of LandauFermi liquid metals. Also relevant to our proposal is the work by Lyo and Plummer [3], which argues that local-field corrections must be included in random-phase approximation (RPA) calculations to correctly reproduce the quasiparticle band shape of Na. With this caveat in mind, in this paper, we show that dynamical electronic correlations hidden in the local, momentum-independent self-energy $\Sigma(\omega)$ play a significant role for the quasiparticle band narrowing and electronic mass enhancement of $\mathrm{Na}$ and related nearly free alkali systems.

On general grounds, for Landau-Fermi liquid metals, the renormalized band structure can be written as $\varepsilon^{\star}(k)=$ $\varepsilon(k) Z \hat{Z}[18]$, where $Z=\left(1-\frac{\partial \Re \Sigma(\omega, k)}{\partial \omega}\right)_{\omega=0, k=k_{F}}^{-1}$ and $\hat{Z}=$ $\left(1+\frac{1}{v_{F}} \frac{\partial \Re \Sigma(\omega, k)}{\partial k}\right)_{\omega=0, k=k_{F}}$. Here, $v_{F}$ and $k_{F}$ are, respectively, the Fermi velocity and Fermi momentum. Since in DMFT the self-energy is momentum independent, the quasiparticle residue $Z$, which defines the renormalized Fermi energy, directly yields the effective mass of quasiparticles [19]: $\frac{m^{\star}}{m}=\frac{1}{Z}=\left(1-\frac{\partial \Re \Sigma(\omega)}{\partial \omega}\right)_{\omega=0}$. Thus, in DMFT, the effective mass ratio $m^{\star} / m$ and $Z$ are both directly related to the slope of $\mathfrak{R} \Sigma(\omega)$, and the many-particle band renormalization is therefore solely given by the product between the bare-band dispersion $\varepsilon(k)$ and quasiparticle amplitude $Z(\omega=0)=Z(0)$.

The possibility of correlated electron physics in purely $p[20,21]$ or $s$ [22] band systems is intriguing, since the naive expectation dictates that the itinerancy (kinetic energy of $p, s$ carriers) is appreciable compared to the electron-electron interactions, as distinct from $d$-band systems, where the $d$ electrons reside in much narrower bands. Thus, understanding the role of electron-electron interactions in materials with active $p$ or $s$ bands is undoubtedly an issue of great contemporary interest. In light of the discussion above, we study how an interplay between appreciable $s$-, $p$-band itinerancy and on-site Coulomb repulsion, $U$, plays a central role in the quasiparticle band narrowing of $\mathrm{Na}$. 


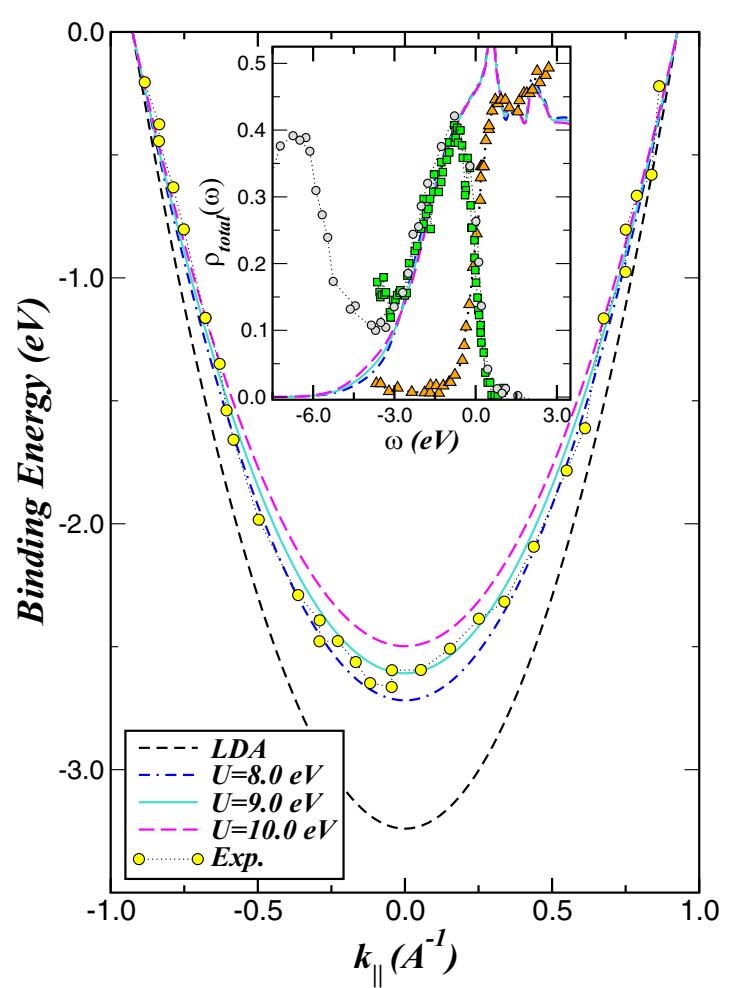

FIG. 1. Comparison between measured (circles) and calculated LDA and LDA+DMFT quasiparticle band dispersion of Na. Good theory-experiment agreement is achieved using $U=9.0 \mathrm{eV}$. Photoemission spectra (circles) and calculated LDA dispersion are taken from Ref. [3]. Inset: Comparison between the LDA+DMFT results for $\mathrm{Na}$ and angle-integrated photoemission (PES, squares and circles) and $\mathrm{x}$-ray absorption spectroscopy (XAS, triangles) taken, respectively, from Refs. [4,10,23]. Good quantitative theory-experiment agreement is visible. In particular, the line shape in PES is accurately resolved in the LDA+DMFT spectrum. Notice as well the high energy satellite in the PES spectrum of Ref. [23], which is not reproduced by LDA+DMFT. (PES data of Ref. [23] was shifted upward by $0.4 \mathrm{eV}$ to coincide with theory and Citrin [10] result.)

\section{THEORY AND RESULTS}

In Fig. 1, we provide a direct comparison between the dispersion curve for the occupied band of $\mathrm{Na}$ obtained from ARPES data [3] measured along $k_{\|}$, the parallel (to the surface) component of the electron momentum [7]. As seen, good theory-experiment agreement for the measured quasiparticle band dispersion $\varepsilon^{\star}(k)=Z_{s}(0) \varepsilon(k)$ (here, $Z_{s}$ is the $3 s$-band quasiparticle amplitude) is obtained using $U=9.0 \mathrm{eV}$ [24] for the correlated multiband, MO problem of $\mathrm{Na}$ (see below). We emphasize, however, that the effective $U$ would be considerably reduced when the many-particle problem of $\mathrm{Na}$ is restricted to a half-field $3 s$ band. In this particular case, an $U=6.0 \mathrm{eV}$ would give quantitive good theoryexperiment agreement (not shown) for the quasiparticle band narrowing and mass enhancement of Na. Below we describe our correlated electronic structure calculations for the manyparticle contribution to the self-energy of $\mathrm{Na}$ arising from local electron-electron interactions.

Theoretical attempts toward a realistic description of good metals in solids involves going beyond traditional zero temperature free-electron gas (FEG) concepts, where $\varepsilon(\mathbf{k})=$ $\frac{\hbar}{2 m} \mathbf{k}^{2}$ and $Z_{0}=1$. With this in mind, and focusing on $\mathrm{Na}$, here we carried out a comprehensive numerical study of electron correlations effects, providing a compelling theoretical evidence of local self-energy corrections to the bare LDA band structure. We use LDA+DMFT [15] to address this long-standing problem of fundamental importance.

Within LDA, the one-electron part of the twochannel model Hamiltonian relevant to $\mathrm{Na}$ is $H^{0}=$ $\sum_{a, \mathbf{k}, \sigma} \varepsilon_{a}(\mathbf{k}) c_{a, \mathbf{k}, \sigma}^{\dagger} c_{a, \mathbf{k}, \sigma}$, where $a$ denotes the $3 s$ and $3 p$ bands crossing $E_{F}$ [1]. Here, $\varepsilon_{a}(\mathbf{k})$ is the two-channel band dispersion, which encodes details of the one-electron (LDA) band structure. These $s$ and $p$ bands are the relevant one-particle inputs for correlated band-structure calculations within LDA+DMFT. In light of non-negligible correlation effects in $\mathrm{Na}$, here we show how a DMFT treatment of many-particle Coulomb interaction effects properly describe the onset of correlated responses seen in the spectral function of Na. This constitutes the one- [25] and MO [15] local interaction terms $H_{s}^{\text {int }}$ and $H_{p}^{\text {int }}$, respectively. The correlated many-body Hamiltonian $H^{\text {int }}=H_{s}^{\text {int }}+H_{p}^{\text {int }}$ considered for $\mathrm{Na}$ thus reads

$$
\begin{aligned}
H^{\mathrm{int}}= & U \sum_{i} n_{s, i, \uparrow} n_{s, i, \downarrow}+U \sum_{i, \alpha} n_{p, i, \alpha, \uparrow} n_{p, i, \alpha, \downarrow} \\
& +U^{\prime} \sum_{p, i, \alpha \neq \beta} n_{p, i, \alpha} n_{p, i, \beta}-J_{H} \sum_{p, i, \alpha \neq \beta} \mathbf{S}_{p, i, \alpha} \cdot \mathbf{S}_{p, i, \beta} .
\end{aligned}
$$

Here $\alpha=x, y, z$ denotes the diagonalized $3 p$ orbitals, $U$ is the on-site Coulomb interaction, $U^{\prime}=U-2 J_{H}$ is the interorbital $p$-band Coulomb interaction term, and $J_{H}$ is the corresponding Hund's coupling. Consistent with earlier studies [26], we focus on the leading local correlation effects and will not consider the pair-hopping term in the MO $p$-band problem of $\mathrm{Na}$ for simplicity [27]. We evaluate the manyparticle Green's functions,

$$
G_{a, \sigma}(\omega, \mathbf{k})=\frac{1}{\omega-\Sigma_{a, \sigma}(\omega)-\varepsilon_{a}(\mathbf{k})},
$$

of the many-body Hamiltonian $H=H^{0}+H^{\text {int }}$ using oneband [16] and MO [17] IPT impurity solvers for $H_{s}^{\text {int }}$ and $H_{p}^{\text {int }}$, respectively.

On general grounds for nonmagnetic electronic systems, the many-particle Green's function of an orbital $\alpha$ can be computed using the following relation for the MO-IPT selfenergy [17]:

$$
\Sigma_{\alpha}(\omega)=\sum_{\beta}\left[N_{\alpha \beta} U_{\alpha \beta}\left\langle n_{\beta}\right\rangle+\frac{A_{\alpha \beta} \Sigma_{\alpha \beta}^{(2)}(\omega)}{1-B_{\alpha \beta} \Sigma_{\alpha \beta}^{(2)}(\omega)}\right],
$$

with $\quad \Sigma_{\alpha \beta}^{(2)}(\omega)=N_{\alpha \beta} \frac{U_{\alpha \beta}^{2}}{\beta^{2}} \sum_{l m} G_{\alpha}^{0}\left(i \omega_{l}\right) G_{\beta}^{0}\left(i \omega_{m}\right) G_{\beta}^{0}\left(i \omega_{l}+\right.$ $i \omega_{m}-i \omega$ ) being the second-order (in $U_{\alpha \beta}$ ) contributions and $N_{\alpha \beta}=2-\delta_{\alpha \beta}$. In contrast to the usual one-band Hubbard model [16], the atomic limit for the MO case contains local, interorbital correlation functions, $D_{\alpha \beta}$ [28]. The equations for the parameters $A_{\alpha \beta}$ and $B_{\alpha \beta}$ are explicit functions of $U_{\alpha \beta}$, $D_{\alpha \beta}$ and the average numbers (per spin $\sigma$ ) $\left\langle n_{\alpha}\right\rangle \equiv n_{\alpha}$ and $\left\langle n_{\alpha}^{0}\right\rangle$ [29]. The latter is the effective number of fermions in the $\alpha$ orbital corresponding to site-excluded Green's function 


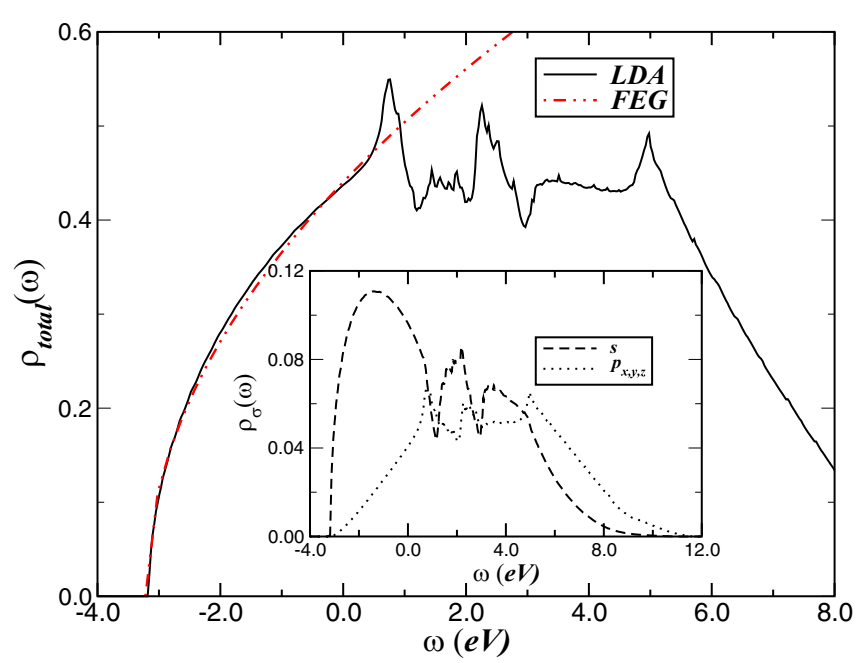

FIG. 2. Comparison between the total LDA DOS of Na (solid line) and the three-dimensional free-electron DOS (dot-dashed line), showing good agreement up to $0.4 \mathrm{eV}$ above $E_{F}$. The inset shows the $3 s$ and $3 p$ DOS used as input to LDA+DMFT. Notice the broad bandwidth in the conduction band states above $E_{F}$.

$G_{\alpha}^{0}(\omega)$ (with $\left[G_{\alpha}^{0}(\omega)\right]^{-1} \equiv\left[G_{\alpha}(\omega)\right]^{-1}+\Sigma_{\alpha}(\omega)$ ). The above relations form a closed set of coupled, nonlinear equations which are solved numerically until convergence is achieved.

It is worth noting here that the IPT ansatz is known to account for the correct low- and high-energy behavior of the one-particle spectral functions and self-energies of oneband [19] and MO models [17,30] in the large dimensional limit (DMFT). It ensures the Mott-Hubbard metal-insulator transition from a correlated metal to a Mott insulator as a function of the Coulomb interaction $U$. The IPT scheme is computationally very efficient, with real frequency output at zero and finite temperatures [31], enabling us to study electronic structure reconstruction of real materials with different orbital character, magnetic, and structural phases.

For the high-symmetry body-centered cubic structure of $\mathrm{Na}$, the $3 s$ and $3 p$ bands crossing $E_{F}$ [1] were obtained using the linear muffin-tin orbital [5] scheme within the LDA [32]. In the inset of Fig. 2, we display the channel-resolved LDA density of states (DOS) used to compute the renormalized LDA+DMFT spectral functions of solid Na. As expected for $s$ and $p$ band systems, the LDA DOS of $\mathrm{Na}$ is broad, with a bare bandwidth of $W \approx 14.5 \mathrm{eV}$. Consistent with early band-structure calculations [1], the width of the valence band is close to $3.2 \mathrm{eV}$. Interestingly as well is the line shape of the total DOS (see main panel) up to $0.4 \mathrm{eV}$ above $E_{F}$ which follows almost perfectly the energy dependence of a threedimensional FEG. However, due to electron-ion interaction effects [33], deviations from this canonical text-book behavior is visible in the conduction band states above $0.4 \mathrm{eV}$. Equally interesting are the deep-hump structures between $1.2 \mathrm{eV}$ and $3.0 \mathrm{eV}$ above $E_{F}$ : As shown in the inset of Fig. 1, these structures are important for understanding the unoccupied band structure [4] of $\mathrm{Na}$ at low energies.

To make progress, in Fig. 3 we show the electronic reconstruction induced by local Coulomb correlation effects in the $3 s$ (main panel) and $3 p$ (inset) band states of $\mathrm{Na}$

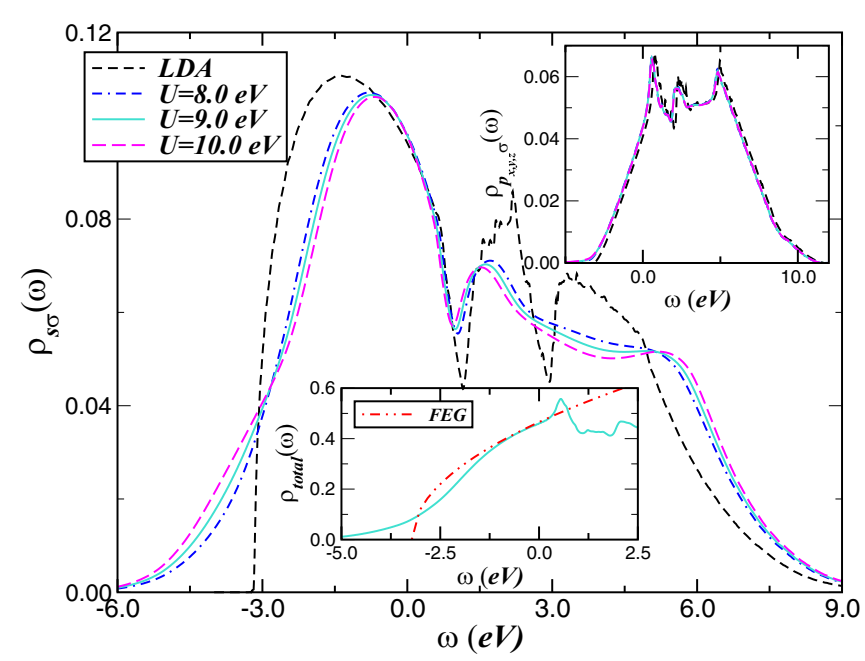

FIG. 3. 3s-band LDA and LDA+DMFT DOS of Na. Notice the large spectral weight transfer from low to high energies and the one-particle band narrowing near $E_{F}$. Top inset displays the corresponding $3 p$-band LDA and LDA+DMFT DOS, showing negligible multiorbital Coulomb correlation effects in this channel with threefold orbital degeneracy. In the low inset, we compare the total LDA + DMFT $(U=9.0 \mathrm{eV})$ DOS with free-electron DOS, showing clear deviations between the two below $1.0 \mathrm{eV}$ binding energy due to correlation-induced dynamical spectral weight transfer.

computed using three different $U$ values and fixed Hund's exchange coupling $J_{H}$. In view of the ambiguity regarding the importance this two-particle exchange interaction to $\mathrm{Na}$ [34], we use $J_{H}=0.5 \mathrm{eV}$ as a model parameter. It is worth noting, however, that the $U$ values considered in this paper are close to the bare Coulomb interaction $U=8.0 \mathrm{eV}$ derived in Ref. [14] and this choice leads to an effective $\left(U_{\text {eff }} \equiv U / W\right)$ Coulomb interaction of $0.55<U_{\text {eff }}<0.69$, implying a weakly correlated scenario for Na metal. Indeed, our fully self-consistent LDA+DMFT(IPT) results for the half-field (one electron per site) electronic configuration of Na shows weak, albeit non-negligible, spectral weight transfer (SWT) across large energy scales. This is a fingerprint of correlated electron systems within DMFT. The dynamical nature of SWT can be characterized by Kondoesque band narrowing at energies near $E_{F}$ and the presence of crossing points in the $3 s$ valance and conduction band at $-2.8 \mathrm{eV}$ and $5.2 \mathrm{eV}$, respectively. Also interesting is the suppression of the $V$ valley at $2.96 \mathrm{eV}$ above $E_{F}$ due large energy dynamical SWT. Moreover, the inset of Fig. 3 shows negligible correlation fingerprints in the $p$-band electronic structure of Na. The lack of SWT in this orbital sector reflects its almost empty nature, and the fact that electron-electron interaction reaches its maximum at half filling and to decrease away from this configuration [35].

Our total LDA+DMFT DOS $\left(\rho_{\text {total }}(\omega)=\right.$ $\left.-\frac{1}{\pi} \sum_{\sigma} \Im\left[G_{s, \sigma}(\omega)+\sum_{\alpha} G_{p, \alpha, \sigma}(\omega)\right], \alpha=x, y, z\right) \quad$ shows qualitative good agreement with $\mathrm{x}$-ray photoemission (PES) $[10,23]$ and K-edge absorption (XAS) [4] data. As seen in the inset of Fig. 1, both PES and XAS lineshapes are correctly reproduced by LDA+DMFT. Particularly interesting are the absorption peaks at $0.7 \mathrm{eV}$ and $2.2 \mathrm{eV}$ in XAS and the PES spectra up to $3.0 \mathrm{eV}$ binding energy, which are correctly 


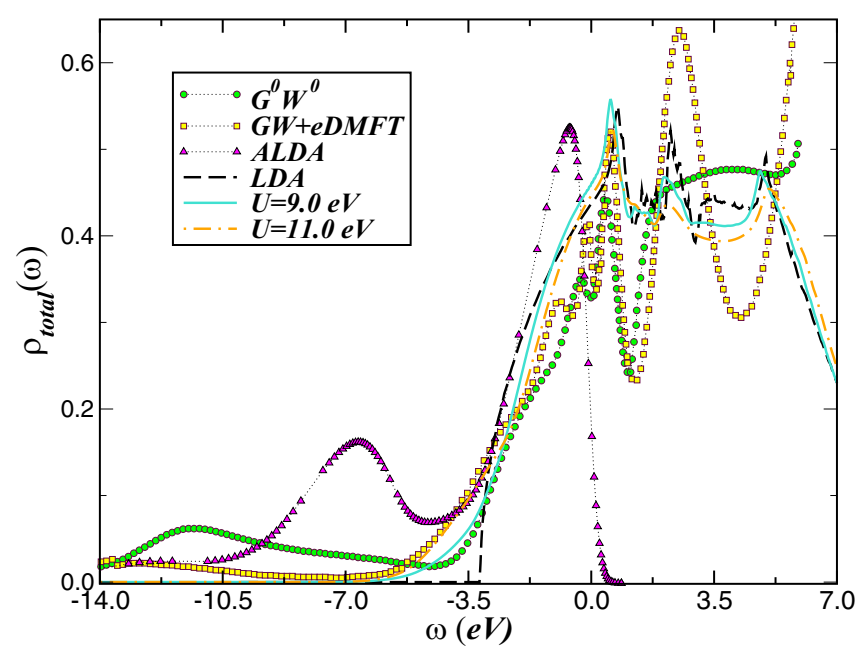

FIG. 4. Total local density of states of $\mathrm{Na}$ metal obtained using LDA, LDA+DMFT $\mathrm{G}^{0} \mathrm{~W}^{0}$, [14], GW+eDMFT [14], and ALDA [13] schemes. Notice the good qualitative agreement between LDA+DMFT, $\mathrm{G}^{0} \mathrm{~W}^{0}$, and GW+eDMFT treatments at low binding energies and the high in energy satellite seen in the $\mathrm{G}^{0} \mathrm{~W}^{0}$ and ALDA results.

reproduced in theory. Taken together, the comparison between (PES and XAS) quasiparticle band narrowing in Fig. 1 and electron mass enhancement testify the use of LDA+DMFT and the validity of the local correction for a consistent explanation of correlation fingerprints seen in the occupied and unoccupied electronic states within the energy range relevant to $\mathrm{AC}$ transport [1] and spectroscopy [2,3,7] experiments.

To obtain detailed information on the evolution of the correlated electronic structure of $\mathrm{Na}$ metal, in Fig. 4 we compare our LDA+DMFT(IPT) total DOS for two $U$ values with results obtained from adiabatic LDA (ALDA) [23], $\mathrm{G}^{0} \mathrm{~W}^{0}$ and combined GW plus extended DMFT (GW+eDMFT) calculations [14]. As seen, good qualitative agreement is obtained using LDA+DMFT(IPT), $\mathrm{G}^{0} \mathrm{~W}^{0}$, and GW+eDMFT implementations from $-5.0 \mathrm{eV}$ binding energy up to $0.8 \mathrm{eV}$ above $E_{F}$. Above this energy, the agreement between LDA+DMFT(IPT) and both $\mathrm{G}^{0} \mathrm{~W}^{0}$ and $\mathrm{GW}+\mathrm{eDMFT}$ treatments is lost. As compared to our LDA result, all correlated total spectral functions show band narrowing at low energies as well as downshifts of the main conduction band peak in LDA at $0.8 \mathrm{eV}$ above $E_{F}$. Also visible is the SWT at the conduction band from low to energies above $3.2 \mathrm{eV}$ binding energy.

To get additional microscopic insights into the correlated electronic structure evolution due to Landau-Fermi liquidness, in Fig. 5 we show the frequency dependence of $3 s$-band self-energy real $[\Re \Sigma(\omega)]$ and imaginary $[\Im \Sigma(\omega)]$ parts. As seen in the main panel of Fig. $5, \Im \Sigma_{s}(\omega)$ follows the $\omega^{2}$ behavior intrinsic to Landau-Fermi liquids [19] at low energies. Low-energy coherence is also reflected in the linear dependence of $\Re \Sigma_{s}(\omega)$ relevant to band mass renormalization seen in experiment [2]. Thus, taken together with earlier studies $[16,17,19]$, our results in Fig. 5 certify the existence of low-temperature Landau-Fermi liquidness in $\mathrm{Na}$ at ambient pressure. However, how stable this coherent many-particle

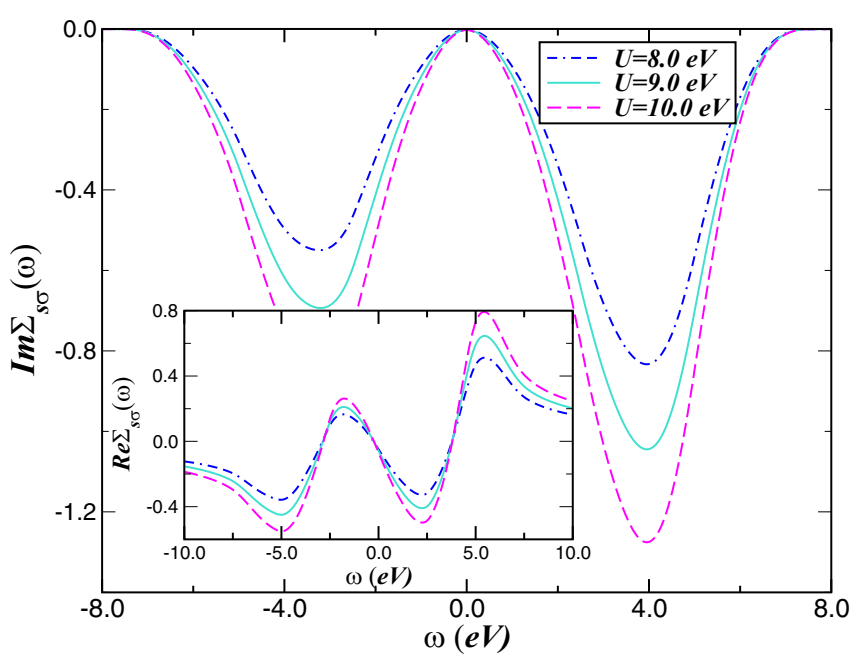

FIG. 5. 3s-band self-energy real (inset) and imaginary (main panel) parts of $\mathrm{Na}$, showing intrinsic Landau-Fermi liquid energydependence within DMFT [19].

state is against nonlocal perturbations $[6,8,13,36]$ not included in $H_{\text {int }}$ or disorder effects [16] remains to be seen. We defer this latter study, however, for a separate work.

As an additional test to our proposal and the validity of the LDA+DMFT approximation to nearly-free metals, in Fig. 6 we compare our results for the $3 s$-band scattering rate $\Gamma_{s}(\omega)=-2 Z_{s}(0) \mathfrak{s} \Sigma_{s}(\omega)$ [37] with results based on FEGRPA calculations of hole and electron line widths taken from Ref. [12]. As expected, both RPA and DMFT results in Fig. 6 show nearly perfect $\omega^{2}$ behavior characteristic of normal Landau-Fermi liquid metals [38]. In spite of different

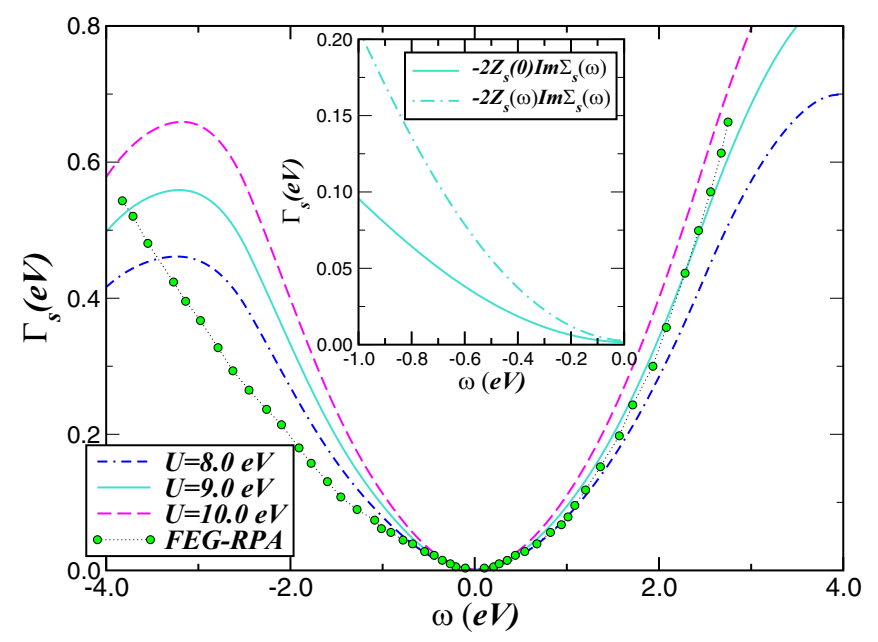

FIG. 6. Comparison between $3 s$ LDA+DMFT and free-electron gas (FEG) RPA calculation for the quasiparticle scattering rate $\Gamma_{s}(\omega)=-2 Z_{s}(O) \Im \Sigma_{s}(\omega)$ (FEG-RPA results are taken from Ref. [12]). Notice the nearly perfect Landau-Fermi liquid behavior and the agreement between RPA and DMFT for electron and hole scattering rates at low energies. Inset shows the changes induced by the energy-dependence in the LDA + DMFT $(U=9.0 \mathrm{eV})$ quasiparticle amplitude $Z_{s}(\omega)$ within the energy window relevant to ARPES experiments. 
formulations and computation frameworks, the good lowenergy FEG-RPA and LDA+DMFT agreement provides support to our model parameter choice. Importantly, and similar to what has been suggested in Ref. [3], below $-0.7 \mathrm{eV}$ the agreement is lost and appreciable deviations are seen at high binding energies, where the RPA quasiparticle scattering rate is considerable smaller as compared to DMFT results. This can be taken as additional evidence for the role played by dynamical, local electron-electron interactions to self-energy corrections inducing $3 s$ electronic reconstruction. Interesting as well is the particle-hole asymmetry found in FEG-RPA, which is well captured by LDA+DMFT, and the nice agreement between FEG-RPA and LDA + DMFT $(U=9.0 \mathrm{eV})$ up to $2.6 \mathrm{eV}$ above $E_{F}$. However, deviations from the highenergy LDA+DMFT response are expected to be seen in future studies, particularly if additional $k$-dependent $[6,8,13,36]$ scattering mechanisms not considered in our description or dynamical screening effects induced by electron-plasmon (electron-boson) interaction [39] are self-consistently taken into account. This many-body corrections to DMFT are predicted to have significant influence on the high-frequency part of the both electron- and holelike scattering rates. However, observation of similar low-energy features as shown in the inset of Fig. 6 in future ARPES experiments would be a stringent test to our Landau-Fermi liquidness scenario and the role played by sizable local electron-electron correlation effects in the electronic structure of $\mathrm{Na}$.

Despite the success of the one-band Hubbard model to capture dynamical many-body properties of correlated electrons [19], including, as shown here, $\mathrm{Na}$ metal, it misses nonlocal Coulomb interaction terms relevant for spin- and charge-density wave instabilities [40] and plasmon physics: [13] The latter has been discussed in the context of valenceelectron excitations [11] and pressure-induced increases in the electron-ion interaction [41] of alkali metals like $\mathrm{Na}, \mathrm{K}$, and $\mathrm{Rb}$. In the case of $\mathrm{Na}$, a plasmonic satellite feature in the experimental spectra is seen around $7 \mathrm{eV}$ binding energy (see the inset of Fig. 1), which is repeated at approximately $-14 \mathrm{eV}$. [23] Interestingly, while good quantitative agreement for the satellite positions was found using ALDA [13] and, to some extent, within $\mathrm{G}^{0} \mathrm{~W}^{0}$ [14], these broad, high-inenergy electronic states are not described using single-site LDA+DMFT as shown here or by GW+eDMFT calculations [14] (see Fig. 4). On general grounds, plasmon excitations and satellites [13] are known to be related to nonlocal interactions between electrons and holes on different bands which can be included via retarded cumulant expansion approximations in LDA calculations [13] or in extended Hubbard models with intersite Coulomb interaction terms [40,42]. We recall that nonlocal interaction terms can be sizable in delocalized broad-band systems [21], with a magnitude reaching up to
$60 \%$ of the on-site Coulomb interaction. Thus, consideration of nonlocal electronic interactions with slow decay with distance will immediately introduce $\mathbf{k}$-dependent corrections to the local DMFT self-energy, which are important for understanding nonlocal scattering mechanisms in ARPES data [37]. Future studies which explicitly include nonlocal contributions $[6,8,40]$ are thus called for to reveal $\mathbf{k}$-dependent self-energy corrections and plasmon satellites arising from electron-plasmon interaction [43] in the high-energy electronic structure $[3,10,14,41]$ of alkali metals.

\section{CONCLUSION}

In summary, we investigated the electronic structure of sodium metal using LDA+DMFT with sizable multi-band, MO interactions. Although the main purpose of this paper is to understand the quasiparticle band narrowing due to electron-electron interactions, a major finding of the present paper is that considerable changes in the electronic structure would be found due to dynamical SWT from low to high energies. Particularly for the $3 s$ conduction band states, where an incipient upper Hubbard band is predicted to be seen at energies close to $5.3 \mathrm{eV}$, this is a clear manifestation that more is different [44] also to nearly-free-electron-like systems. Our Landau-Fermi liquidness approach suggests that retarded interactions stemming from collective plasmon excitations [39] play a minor role in determining the frequency dependence of quasiparticle (electron/hole) scattering rates at low energies. Future collective charge excitation studies of correlated electrons are called for to corroborate this prediction for $\mathrm{Na}$ metal.

\section{ACKNOWLEDGMENTS}

L.C.'s work is supported by CNPq (Grant No. 304035/2017-3). Acknowledgment (L.C.) is also made to the Institute for Solid State Research at IFW Dresden for hospitality and to Jörg Fink for useful and enlightening discussions on the quasiparticle band narrowing of sodium metal. S.L. acknowledges support from the UK Research Council for using work in the paper that was undertaken by a student under Project No. EP/M50631X/1 as well as the DFG for support under the priority Project No. SPP1415 and for a personal Heisenberg grant. S.L. also thanks ARCCA Cardiff for computational resources. Via S.L.'s membership in the UK's HPC Materials Chemistry Consortium, which is funded by EPSRC (No. EP/L000202), this work made use of the facilities of ARCHER, the UK's National High-Performance Computing Service, which is funded by the Office of Science and Technology through EPSRC's High End Computing Programme.
[1] See W. Y. Ching and J. Callaway, Phys. Rev. B 11, 1324 (1975) and references therein.

[2] E. Jensen and E. W. Plummer, Phys. Rev. Lett. 55, 1912 (1985).

[3] I.-W. Lyo and E. W. Plummer, Phys. Rev. Lett. 60, 1558 (1988).

[4] P. H. Citrin, G. K. Wertheim, T. Hashizume, F. Sette, A. A. MacDowell, and F. Comin, Phys. Rev. Lett. 61, 1021 (1988).
[5] O. K. Andersen, Phys. Rev. B 12, 3060 (1975).

[6] H. Yasuhara, S. Yoshinaga, and M. Higuchi, Phys. Rev. Lett. 83, 3250 (1999).

[7] Kenneth W.-K. Shung, Phys. Rev. B 44, 13112(R) (1991).

[8] J. Lischner, T. Bazhirov, A. H. MacDonald, M. L. Cohen, and S. G. Louie, Phys. Rev. B 89, 081108(R) (2014). 
[9] L. Hedin and S. Lundqvist, Solid State Phys. 23, 1 (1969); X. Zhu and A. W. Overhauser, Phys. Rev. B 33, 925 (1986); J. E. Northrup, M. S. Hybertsen, and S. G. Louie, Phys. Rev. Lett. 59, 819 (1987); Phys. Rev. B 39, 8198 (1989).

[10] P. H. Citrin, Phys. Rev. B 8, 5545 (1973).

[11] A. vom Felde, J. Sprösser-Prou, and J. Fink, Phys. Rev. B 40, 10181 (1989).

[12] J. S. Dolado, V. M. Silkin, M. A. Cazalilla, A. Rubio, and P. M. Echenique, Phys. Rev. B 64, 195128 (2001).

[13] J. S. Zhou, M. Gatti, J. J. Kas, J. J. Rehr, and L. Reining, Phys. Rev. B 97, 035137 (2018).

[14] F. Nilsson, L. Boehnke, P. Werner, and F. Aryasetiawan, Phys. Rev. Mater. 1, 043803 (2017).

[15] G. Kotliar, S. Y. Savrasov, K. Haule, V. S. Oudovenko, O. Parcollet, and C. A. Marianetti, Rev. Mod. Phys. 78, 865 (2006).

[16] M. S. Laad, L. Craco, and E. Müller-Hartmann, Phys. Rev. B 64, 195114 (2001).

[17] L. Craco, Phys. Rev. B 77, 125122 (2008).

[18] C. M. Varma, Z. Nussinov, and W. wan Saarloos, Phys. Rep. 361, 267 (2002).

[19] A. Georges, G. Kotliar, W. Krauth, and M. J. Rozenberg, Rev. Mod. Phys. 68, 13 (1996).

[20] R. Bhandari and L. M. Falicov, J. Phys. C: Solid State Phys. 6, 479 (1973); A. J. R. da Silva and L. M. Falicov, Phys. Rev. B 52, 2325 (1995); J. A. Chan, S. Lany, and A. Zunger, Phys. Rev. Lett. 103, 016404 (2009); J. Winterlik, G. H. Fecher, C. A. Jenkins, C. Felser, C. Muhle, K. Doll, M. Jansen, L. M. Sandratskii, and J. Kubler, ibid. 102, 016401 (2009); L. Craco, M. S. Laad, and S. Leoni, Sci. Rep. 7, 2632 (2017).

[21] T. O. Wehling, E. Şaşığlu, C. Friedrich, A. I. Lichtenstein, M. I. Katsnelson, and S. Blügel, Phys. Rev. Lett. 106, 236805 (2011).

[22] G. Chiappe, E. Louis, E. San Fabián, and J. A. Verges, Phys. Rev. B 75, 195104 (2007); G. Stutz, F. Wohlert, A. Kaprolat, W. Schülke, Y. Sakurai, Y. Tanaka, M. Ito, H. Kawata, N. Shiotani, S. Kaprzyk, and A. Bansil, ibid. 60, 7099 (1999).

[23] H. Höchst, P. Steiner, and S. Hüfner, Z. Phys. B: Condens. Matter 30, 145 (1978).

[24] Within LDA+DMFT, we obtain $Z_{s}(0)=0.806$, and, thus, a bandwidth of $2.61 \mathrm{eV}$ (19.2\% narrowing) consistent with $2.65 \pm 0.05 \mathrm{eV}$ seen in experiment [3]. We find the effective band mass renormalization $\frac{m^{\star}}{m}=1.24$ is also in good accord with experimental determined values $1.23<\frac{m^{\star}}{m}<1.28$ [[3], [2]].

[25] J. Hubbard, Proc. Roy. Soc. London A 276, 238 (1963).

[26] K. Held and D. Vollhardt, Eur. Phys. J. B 5, 473 (1998); R. Yu, J.-X. Zhu, and Q. Si, Phys. Rev. Lett. 106, 186401 (2011); G.
Liu, N. Kaushal, S. Li, C. B. Bishop, Y. Wang, S. Johnston, G. Alvarez, A. Moreo, and E. Dagotto, Phys. Rev. E 93, 063313 (2016).

[27] We have not consider the interband Coulomb interaction term, $U_{s p}$, between $s$ and $p$ electrons in $H^{\text {int }}$ for simplicity.

[28] P. Pou, R. Pérez, F. Flores, A. Levy Yeyati, A. Martin-Rodero, J. M. Blanco, F. J. García-Vidal, and J. Ortega, Phys. Rev. B 62, 4309 (2000).

[29] L. Craco, M. S. Laad, and E. Müller-Hartmann, Phys. Rev. Lett. 90, 237203 (2003).

[30] N. Dasari, W. R. Mondal, P. Zhang, J. Moreno, M. Jarrell, and N. S. Vidhyadhiraja, Eur. Phys. J. B 89, 202 (2016).

[31] M. S. Laad, L. Craco, and E. Müller-Hartmann, Phys. Rev. B 73, 045109 (2006).

[32] LDA self-consistent solutions for Na were reached by performing calculations with 624 irreducible k-points. The radii of the atomic spheres were chosen as $r=2.12$ a.u. to minimize their overlap.

[33] M. J. G. Lee, Phys. Rev. 178, 953 (1969).

[34] F. Masnou-Seeuws and R. McCarroll, J. Phys. B: Atom. Molec. Phys. 7, 2230 (1974).

[35] L. de Medici, G. Giovannetti, and M. Capone, Phys. Rev. Lett. 112, 177001 (2014).

[36] E. V. Chulkov, A. G. Borisov, J. P. Gauyacq, D. Sánchez-Portal, V. M. Silkin, V. P. Zhukov, and P. M. Echenique, Chem. Rev. 106, 4160 (2006).

[37] See J. Fink, J. Nayak, E. D. L. Rienks, J. Bannies, S. Wurmehl, S. Aswartham, I. Morozov, R. Kappenberger, M. A. ElGhazali, L. Craco, H. Rosner, C. Felser, and B. Büchner, Phys. Rev. B 99, 245156 (2019).

[38] J. J. Quinm and R. A. Ferrel, Phys. Rev. 112, 812 (1958); see also G. D. Mahan, Many-Particle Physics (Kluwer Academic, Dordrecht, 2000).

[39] M. Casula, A. Rubtsov, and S. Biermann, Phys. Rev. B 85, 035115 (2012).

[40] G. Rohringer, H. Hafermann, A. Toschi, A. A. Katanin, A. E. Antipov, M. I. Katsnelson, A. I. Lichtenstein, A. N. Rubtsov, and K. Held, Rev. Mod. Phys. 90, 025003 (2018).

[41] I. Loa, K. Syassen, G. Monaco, G. Vankó, M. Krisch, and M. Hanfland, Phys. Rev. Lett. 107, 086402 (2011); J. IbañezAzpiroz, B. Rousseau, A. Eiguren, and A. Bergara, Phys. Rev. B 89, 085102 (2014).

[42] L. Craco, M. S. Laad, S. Leoni, and A. S. de Arruda, Phys. Rev. B 87, 155109 (2013); R. C. Juliano, A. S. de Arruda, and L. Craco, Solid State Comm. 227, 51 (2016).

[43] D. Vigil-Fowler, S. G. Louie, and J. Lischner, Phys. Rev. B 93, 235446 (2016).

[44] P. W. Anderson, Science 177, 393 (1972). 